\title{
The Status of Prevention in the Education of Child Psychiatrists
}

\author{
Saul I. Harrison, MD \\ University of Michigan Medical Center \\ James G. Delano, MD \\ Mayo Clinic
}

ABSTRACT: While the pioneers of modern child psychiatry focused much of their attention on prevention, the situation today is markedly different. A questionnaire survey of child psychiatric residency programs suggests that when attention is devoted to prevention in the education of child psychiatrists, it is often ambiguous, haphazard, and minimal. This impelled the authors to sample the current status of available knowledge about preventive child psychiatry with an assessment of the validity of the knowledge and its potential utility. These are followed by some suggestions for child psychiatric education.

Although a variety of factors contributing to the development of child psychiatry have been identified $[1,2,3,4]$, there is remarkable unanimity regarding the seminal role of the American Child Guidance Clinic movement. That the Child Guidance Clinic's original aim was the prevention of delinquency is often forgotten because this explicitly limited goal shifted to the more global one of psychiatric care for all children regardless of age and type of psychiatric difficulty. It could be argued that this shift from the particular to the general is only apparent, and that in fact the original preventive rationale persisted because it was thought, albeit without supportive evidence, that

Dr. Harrison is Professor of Psychiatry and Director of Child and Adolescent Psychiatric Education, University of Michigan Medical Center. Reprint queries should be directed to Dr. Harrison at Children's Psychiatric Hospital, University of Michigan Medical Center, Ann Arbor, Michigan 48109. Dr. Delano is Senior Consultant in Child and Adolescent Psychiatry, Mayo Clinic, Rochester, Minnesota. The authors appreciate the advice and collaboration of the American Academy of Child Psychiatry's Committee on Prevention. Drs. Alan Levy and Melvin Shapiro were particularly helpful. The editorial advice offered by Jacqueline Moore is also appreciated. 
children's psychiatric disorders were more amenable to treatment, thereby minimizing or eliminating later consequences. Nevertheless, since the burgeoning of child guidance clinics in the 1920s, prevention has remained a largely unspoken aim of child psychiatry, an assumption that has received too little articulation or scrutiny.

Interestingly, for many of us ontogeny seems to recapitulate phylogeny in that the process of becoming a child psychiatrist involves individual developmental changes paralleling the historical evolution of the field at large. Many physicians who wish to become child psychiatrists are initially attracted to the field by a desire to prevent psychiatric problems. The process of learning psychiatry, however, somehow diminishes the desire to prevent problems. Perhaps this is a consequence of perceiving the challenges as so overwhelming that in the interest of self-protection we retreat, so to speak, to clinical work (which we subsequently learn can be designated as tertiary prevention). Problem solving generally appears to be less risky than planning; thus we find greater security in the easier task of defining what to treat than in delineating what to prevent.

Over the years advances in knowledge have resulted in changes in our conceptions about children and their problems, which in turn have led to changes in the structures of child psychiatric services. These services have assumed different forms in a variety of settings. Concomitantly, standards and procedures for education and certification of child psychiatrists have been developed and applied. Nevertheless, the description of the basic content of a child psychiatry residency curriculum emanating from the 1963 Conference on Training in Child Psychiatry [5: pp. 61, 74] gave scant attention to prevention, although the report had designated it as "the ultimate goal" of the child psychiatrist. Clearly, prevention was not delinated as one of the five major functions (diagnosis and treatment, collaboration and consultation, administration, research, and teaching) of the child psychiatrist for which training was deemed necessary. In the eyes of some of the beholders of that report, however, there are suggestions that prevention was assumed to be implicitly ubiquitous, even if amorphous and between the lines, in all facets of child psychiatric education.

In the ensuing decade, the status of prevention in child psychiatric residency education has not become any more clearly defined despite other signs of increasing concern about the need for prevention. To the best of our knowledge, prevention has never been a sustained focus in national meetings of residency program directors or in reviews of residency programs by colleagues in the service of various accrediting and funding agencies. Exemplifying this state of affairs, the com- 
prehensive 1974 survey of training programs in child psychiatry, undertaken by the Committee on Certification in Child Psychiatry of the American Board of Psychiatry and Neurology, did not explicitly include "prevention" among the several categories listed on the questionnaire directed to program directors (although it is possible that prevention was implicitly subsumed under other listed headings such as "child development," "crisis intervention," "consultation skills," "legal aspects of child psychiatry," and "other").

\section{Questionnaire}

In this climate, the American Academy of Child Psychiatry's Committee on Prevention decided to solicit information by means of a questionnaire addressed to child psychiatry residency programs. In doing so, there was no pretense that a comprehensive survey using a rigorous methodology was being conducted. On the contrary, the committee informally characterized the quest as a "fishing expedition," based on the justification that whatever information was "fished out" would represent an advance by offering a glimpse of the extent and content of either actual or idealized educational efforts in the area of prevention (whether designated as such or under other headings such as "child development," "community," "public health," "consultation").

The 31 responses (primarily from university programs) received from 115 programs to whom questionnaires were addressed represent a low return rate. Nevertheless, considering the open-ended nature of the inquiry into an issue about which there were practically no data, the answers proved to be of interest, if viewed as a nonstatistical sampling. The responses ranged from perfunctory, to informative, to extensively thoughtful; some seemed despairing, apologetic, or cynical, while others were confident, enthusiastic, and even entrepreneurial.

Consistent with the assumption that prevention is intertwined in all aspects of child psychiatry, one respondent asserted that to describe their program's teaching of prevention would require a description of the entire educational program. Another respondent did not explicitly state it but appeared to have presented an entire residency program, and indeed this proved to be the most integrated response of all received.

\section{Teaching of Prevention Designated as Such}

The fact that programs rarely reported activities explicitly labeled as "prevention" may have been influenced by the structure of the 
questionnaire, which inquired first about the teaching of prevention under other labels. An array of activities were reported that could easily be subsumed under a preventive heading: genetic counseling; focus on siblings in case evaluations; study of early.mother-infant interactions with indicated intervention; crisis intervention with highrisk groups such as prematures, the disadvantaged, the physically handicapped; Head Start; "problem center" at a housing project; daycare programs for infants and toddlers from unsettled homes; nutrition in mothers and infants; early maladaptive or symptomatic behaviors; focusing on specific critical developmental phases, such as mother-infant pairs in home visits and the 2nd year of life; classroom management; special education procedures; early detection of psychological problems in schools; maternal depression; psychology of parenting; drug abuse education; community hotlines.

\section{Prevention Taught Under Other Labels}

Almost every program stated that prevention was taught under the headings of "child development" and "community consultation." This was consistent with the view of the Conference on Training in Child Psychiatry [5], which identified child development as one of three major basic concepts. Indeed, one of the few references to prevention that appeared in the report of that conference was in relation to child development: "Prevention and treatment always [have] as a goal the resumption and continuation of satisfactory developmental progress." The frequency with which consultation was noted by our respondents was consistent also with Caplan's [6] emphasis on mental health consultation in his discussion of the principles of prevention. Most of the consultative efforts cited by our respondents were with schools and pediatricians. Less frequently mentioned were public health nurses, well-baby clinics, family agencies, police, and clergy.

Other than "child advocacy," the additional headings under which prevention was noted as being taught seemed to be routine aspects of residency education such as literature, supervision, and various kinds of clinical activity.

\section{Suggestions for Ideal Programs}

The most frequent references to primary prevention appeared in response to the questionnaire's explicit request for suggestions for ideal programs. One respondent emphasized the importance of offering residents opportunities to be innovative and creative, particularly 
in areas involving different population groups and different kinds of problems.

Another respondent outlined a detailed proposal that included the following elements: (a) study of child development, parent-child interaction, and family study; (b) infant nursery program; (c) longitudinal study of normal infants; (d) observations in nursery school and Head Start programs; (e) identification of populations at risk; (f) follow-up study with prediction of normal family, at-risk family, and family showing early deviance.

Other responses emphasized the desirability of a rich background in consultation, familiarity with all socioeconomic groups, and an awareness of an involvement in critical social areas, such as medical care delivery systems, drugs, public welfare programs, housing, job training, family planning, and prenatal clinics.

\section{Additional Commentary from Respondents}

Several respondents commented that upon receiving the questionnaire the attention of their program was directed to this neglected area. As a consequence, formal academic programs for residents regarding the theory and practice of prevention were considered. It should be noted also that committee members were told informally by representatives of programs that had neglected to return a completed questionnaire that it had served a similar purpose in their programs. Since then, we have not been informed of any tangible results of these reappraisals, nor have we inquired formally.

Sharply contrasting with these positively oriented, nonspecific global comments is the specific comment by one respondent that he had watched this "movement at close range since its invention" and found little in it except obfuscation. In this respondent's experience, prevention is not only a concept with minimal utility but, in practice, it has proved to be a definite problem. This respondent went on to assert that prevention might well serve as a useful framework for public health, but its use in psychiatry has been unfortunate.

The respondent who had said that to describe the teaching of prevention in his program would require an outline of the entire residency advised that teaching epidemiology and primary prevention is not possible unless the residents are well grounded in child development, which this answer described as the most difficult area in which to sustain residents' interest, despite the fact that development is talked about so much. The respondent added that, while primary prevention is an area in need of the highest and most refined scientific 
sophistication, it fails to "turn on" clinically minded residents or clinically minded faculty. Questioning whether primary prevention is actually being taught in any program or is essential in the education of clinical psychiatrists, this respondent suggested that primary prevention might appropriately become a sub-subspecialty suitable for advanced residency education.

Another perspective regarding primary prevention came from a respondent who stated initially that an increasing proportion of his program's clinical, educational, and research efforts was directed to primary prevention; however, he added that "inertia is a major obstacle." Furthermore, this respondent noted that statistically minded clinician-researchers tend to "demean primary prevention as unprovable."

If the authors of this paper were to exercise editorial license, we would change the respondent's term "unprovable" to a phrase about the "extreme complexity of the methodology required to demonstrate the effectiveness of preventive programs." Then we would be tempted to expand our license by questioning the extent to which "inertia" might in fact stem from "disapproval" expressed by the respondent's research-minded colleagues and, indeed, what effect this might have on the teaching of prevention in university settings, where faculty promotion is so frequently dependent on the quantity of one's visible research productivity.

\section{Significance of These Responses}

This nonsystematic glimpse of some of the highlights of responses to the questionnaire suggests that in some residency programs there are elements of the curriculum in which prevention is implicitly and at times explicitly an aspect. The issues of the effectiveness and viability of the educational efforts are far more uncertain than its visibility. Although the answers to our specific questions failed to clarify the visibility issue, the frequency with which words such as "oriented," "filter," and "woven" were employed probably says something about its visibility and clearly about its lack of primacy.

Some thought that the ideal residency program suggested by the 1963 conference was utopian. Nevertheless, an approximation of such a program has probably been achieved in many centers-both prior to and since that conference. Although many believe that the seminal ideas for the outline of an ideal preventive program have long been available, for example, as articulated by Caplan [7], the issue of its integration in child psychiatric residency programs remains uncer- 
tain. As a first step, it would seem desirable that the current ambiguous status of education in prevention be clarified. The next step is less certain. Should education in prevention be unequivocally dismissed? Or should decided efforts be made to teach prevention as a significant function of a child psychiatrist? Should education in prevention permeate and penetrate the entire residency program as Anthony [8] suggested for research? Or should it be a clearly demarcated, sequestered, easily identified part of the training of every child psychiatrist? If so, how large a part should it be? Also, what do we know about preventive child psychiatry and with what degree of certainty? The remainder of this report will constitute an expository formulation of our perception of the current state of knowledge about prevention in child psychiatry on which residency programs could base educational efforts.

\section{Knowledge About Prevention}

Before we sample some of the significant aspects of preventive child psychiatry, the vital conceptual distinction between the promotion of mental health and the prevention of mental illness should be emphasized. The two are not identical although they sometimes overlap. It should be noted also that it is unlikely that the central importance of this distinction reflects deficiencies in the medical model. This is not stated with the intent of defending the medical model; it is simply an effort to assert that it is our impression that similar distinctions between these concepts and processes would be vital in the context of whatever model is employed.

\section{Knowledge at the Top Level of Certainty}

There is no challenge to the proposition that we know how to prevent psychopathology caused by certain biological problems such as phenylketonuria, galactosemia, cretinism, tertiary syphilis, pellagra, and protein deficiency during pregnancy and infancy.

The fact that psychopathology resulting from these biological difficulties is not always prevented does not reflect uncertainty about our knowledge so much as it represents problems in applying that knowledge. Needless to say, this is hardly unique to our field as it pertains also to fluorides, seat belts, cigarettes, pollution, war, and so on.

Unfortunately, this brief statement represents the state of our knowledge at this highest level of certainty. Of course, it is easy to 
be concise when one knows the subject matter as evidenced by those Nobel Prize-winning papers that have not occupied much more than a page or two. Therefore, stating what is known about prevention with less certainty than the foregoing will require much more space.

\section{Knowledge Supported by Convincing but Challengeable Presumptive Evidence}

Exemplifying the second level of confidence are factors like the persuasive but challengeable notion that family planning can promote the ideal state of affairs where "every child is a wanted child." Although being wanted at birth is no guarantee of mental health, there is considerable evidence suggesting that if a child is born unwanted, he is potentially vulnerable and therefore runs a greater risk of developing emotional difficulties. The evidence is sufficiently persuasive to convince many that allowing an unwanted baby to be brought into this world is comparable to allowing a baby to remain unvaccinated against smallpox. Obviously the analogy is less than perfect from the preventive perspective. Nevertheless, it is cited in view of the fact that there is a minority of people who deeply believe compulsory vaccination to be wrong and indeed sinful. In terms of numbers they are relatively insignificant; however, they are worth considering in the context of the (quantitatively more significant) controversy that is sometimes generated by alluding to the preventive psychiatric potential of contraception and abortion. Referring to its preventive psychiatric value neglects the "Right to Life" moral view of abortion as sinful and criminal, while implicitly reinforcing the "Planned Parenthood" stance that considers not having an abortion and bringing a baby that no one wants into the world as being morally wrong. While recognizing the plurality of moralities, the issue under discussion here is preventive psychiatry.

From this controversial issue, let us go further and spin a fantasy that may deserve the courage of our convictions. Given the unassailable proposition that a firm sense of reality is important for mental health, should we not seriously consider the fact that licensure for marriage is often a farce in today's world and that "licensure" to conceive a child might be more relevant in the preventive psychiatric context? Margaret Mead [9] has addressed this by suggesting two types of marriage: (a) individual marriage, which essentially is publicly sanctioned cohabitation; and (b) parental marriage, which is defined by its title and which would be more difficult to enter and to dissolve. Mead emphasizes that this proposal does not entail the con- 
cept of a trial marriage, which is a logical paradox to the extent that it is impossible to try a commitment without actually making the commitment.

An additional and far more radical approach to the same issue would be a proposal to immunize all babies against conception, and then to de-immunize them when either one or two people request such de-immunization when in a rational frame of mind, making the criteria for granting such requests comparable to the criteria now used to grant marriage licenses.

In asserting this bold suggestion, which may seem wild at first, it should be emphasized that there is absolutely no intention to limit freedom of choice, nor to give weight to Torrey's [10] concern that preventive psychiatry inexorably leads toward "psychiatric fascism." On the contrary, our intention is to enhance truly free and rational decision making. In other words, de-immunization would force people to make a decision about conception (which should be distinguished from a decision about sex or commitment or marriage) while vertical in the light of day instead of under horizontal circumstances that might interfere with serious consideration of long-term consequences.

A second example of an issue about which the evidence is persuasive but challengeable pertains to the value of consistent relationships in infancy and the effects of emotional deprivation, demonstrated by Spitz [11], Bowlby [12], Goldfarb [13], and others. The practical effects of these data on changing certain child-caring practices in the Western world have been considerable-for example, changes in adoptive procedures, so that adoptions now tend to occur in the neonatal period, eliminating the several months' delay that used to be routine; obstetrical rooming-in; liberalized visiting in pediatric inpatient settings; the preponderance of foster homes, representing a shift from the traditional institutional orphanage-type settings.

The consequences of these progressive changes have been manifold, and new problems have emerged such as those resulting from foster placement. Without meaning to minimize the seriousness of these new problems, it should be noted that they do not necessarily mean that the shift of public child rearing from institutional settings to foster homes was not a mark of progress. We are all familiar with the unintended negative consequences of some scientific and technological achievements. For example, the effectiveness of antibiotics has led to the development of tough, resistant strains of organisms; the increasing longevity of the human life span has resulted in an increased incidence of degenerative diseases and malignancy. 
Examples of other problems derived from success, more closely related to preventive child psychiatry, might be the reports of those unanticipated effects stemming from the technology that has enabled more premature babies to survive. For example, Call [14] has suggested that premature infants demonstrate psychological attachment and dependence on the equipment to which they have been attached, thereby requiring gradual withdrawal resembling a weaning process. Of greater obvious significance are the accumulating observations (see Klaus and Kennell's [15] summary) of what appears to be anticipatory grief on the part of parents of prematures that appears to interfere with development of affectional bonds. Logging the visits and calls by parents to the premature nursery suggests that less than two per week is correlated with a high incidence of subsequent "parenting disorders," such as failure to thrive and perhaps child abuse. In consequence, there have been changes in premature nursery routines so as to minimize the mother-infant separation by facilitating and encouraging parental participation in premature nursery care. The traditional concerns about contamination have been dissipated by observation of the washing and gowning practices employed by the various attendants. As might be predicted, parents were far more thorough, careful, and meticulous than were physicians.

Another example of problems generated by success, on the level of convincing but challengeable presumptive evidence, is suggested by Pasamanick and Knobloch's [16] hypothesis that the reduction of mortality from prematurity and fetal death produces an increase in marginal survivors, specifically those with cerebral dysfunction. Pasamanick and Knobloch's concept of a continuum of reproductive causality is founded on an epidemiological correlation of a wide spectrum of neuropsychiatric disabilities (e.g., cerebral palsy, mental retardation, epilepsy, behavior disorders, reading disabilities) with obstetrical complications and with socioeconomic status. The evidence suggests that the disadvantaged share an increased vulnerability to a cyclical developmental sequence of despair that encompasses: (a) higher incidence of pregnancy wastage; (b) more birth defects and brain dysfunction; (c) increased exposure to adverse environmental influences, such as injuries, poison, undernutrition, and neglect; (d) language and cognitive difficulties; (e) school maladjustment; (f) academic deficiencies; (g) delinquency; (h) early pregnancy; (i) school dropout; (j) unemployment; (k) major social problems, such as addiction, crime, and violence; (l) despair; and (m) repetition of cycle.

Additional support for this kind of thinking may be found in From Birth to Seven [17], a publication of the British National Child De- 
velopment Study. It reports the results of follow-up at age 7 of the 16,000 children born in England, Scotland, and Wales during a 1week span in 1958. Dividing them into five social strata demonstrated that the lower social strata suffered in speech development, physical growth, and academic performance. By age 7, for example, there was a 17-month difference in reading levels between the two highest social classes and the lowest. The study points to factors readily observable during the 1st week of life suggesting the predictability of vulnerability to physical and mental handicaps. Among these factors are an unwed mother, unskilled father, high birth rank (fifth of later child), abnormal delivery, prematurity or postmaturity (defined in this study as less than 37 weeks or more than 42 weeks), and illness during the 1st week of life. On these bases, one-quarter of all the infants were identified as "high risk," enabling prediction of more than half the subsequently handicapped children. Additional research endeavoring to identify clusters of interacting variables enabling specificity of predictions would be highly desirable; nevertheless, postponing intervention pending such important information would seem unfortunate for many of these children.

The obvious difficulties in sorting out the genetic-biological and sociocultural determinants of the foregoing data contribute to major controversies regarding the utility of such knowledge. Because it is recognized that our knowledge regarding extent to which nature and/ or nurture contribute to these problems is far less than certain, there is a tendency to focus on philosophical-political issues rather than on empirical ones. Restraining the debate within what are hopefully professional and scientific bounds tends to focus it on the utility of the knowledge that is available. One side asserts that methodological flaws have resulted in such challengeable information that any action based on such information must be at considerable risk of failure. Another point of view asserts that, without intending to minimize the potential value of additional research because new and better knowledge would be welcome, there nevertheless is no basis on which to justify additional delay in the social application of what is known. This results in widely diverging preventive proposals. One polar extreme, using a variant of genetic reasoning that might be characterized also as social Darwinism (although some assert it is racism in a scientific guise), suggests devising means of encouraging selective breeding, such as offering subsidies to vulnerable groups for not breeding. The opposing camp announces that our knowledge to date gives us an opportunity to identify high-risk populations and individuals for whom enrichment programs are indicated to minimize vul- 
nerability. The possibilities of this secondary preventive approach are so wide ranging that even something of the magnitude of the report of the Joint Commission on the Mental Health of Children [18] had been criticized by some because of insufficient comprehensiveness.

Consistent with the style of this presentation, we will highlight only a few of the many possible social applications that could have preventive impact. Of central significance is nutrition and the distribution of prenatal and related medical services during pregnancy and childhood. Related to the nutritional issue is the problem posed by television advertising that endeavors to influence young children to want sweet-tasting, high-calorie food of low nutritional value. Following either the nutritional theme or the advertising theme could introduce a host of other possibilities, for example, the influence of television advertising on making the use of drugs appear like an attractive, instant solution to anything unpleasant.

Another vital consideration that should be stressed is the issue of educational enrichment, for example, the controversy as to whether programs like Head Start were useless from the long-range point or view, or whether they were too little, too late, and too brief. Toward the other end of the educational continuum, there is the possibility of identifying potential school dropouts and offering them programs in their long-range educational and vocational interest, permitting "dropping out within the educational system."

Clinicians generally agree that the diagnosis of an emotional disorder suggests that others at home may also be vulnerable, if not disturbed. Indeed, instances have been reported in which a family "sends" its least disturbed member as an "emissary" to the clinic. Yet, in clinical settings that do not routinely employ a family approach, clinicians are often overextended in their efforts to respond to those individuals who seek and urgently need their services, thereby leaving unanswered the question of responsibility for the possible additional vulnerability or disturbance in the patient's family. Some have compared the situation to that of suspecting unvaccinated children at home. Unfortunately, clinicians who are closest to such situations are often forced to appear the most resistant about following up on their concerns because of factors that have far less to do with their clinical judgment than their concerns about diluting the clinic's already limited abilities.

A response to the aforementioned vaccination analogy is often presented as the choice between quantity or quality as represented by the dilemma posed by an epidemic in which there is a shortage of antibiotics. The choice is between an adequate dosage for small num- 
bers of people (while trying to avert future shortages) against diluting the small current supply of antibiotics in huge cauldrons of chicken soup and distributing inadequate doses of antibiotics in comforting dishes of chicken soup. The authors' ambivalence about this issue leads to another story about the two fishermen who see someone calling for help, floating downstream. They rescue the person, but shortly after returning to their fishing they are interrupted by a similar drowning person. When this happens a third time, instead of heading toward the water, one of the fishermen starts to run along the shore and responds to an accusatory question from his partner about deserting the drowning individual in the river by saying that he is going upstream to get the so-and-so who is pushing all the nonswimmers into the water.

Such ambivalence will not be resolved by recounting additional allegories. Perhaps the means of dealing effectively with it is not readily evident because we are too limited to an individualistic frame of reference, as is revealed by our choice of analogies. After all, both vaccination and antibiotics are individualistic approaches despite their effective mass applicability. Yet McDermott [19] asserted that despite the significance of these scientific advances, they were probably outdistanced by far, in terms of health for the greatest number, by the historical shift to eating food on tables instead of on the floor. Is it the case that entirely new ways of thinking, new concepts, and totally new approaches will enable us to resolve our ambivalence?

At our current level of knowledge and conceptualization, the debate can be expressed nonallegorically by noting the primary preventive value of identifying vulnerable "high-risk" populations for whom programs designed to prevent difficulties can be established. From the secondary preventive perspective, there is the advantage of early case finding, facilitating early intervention and thereby enhancing effectiveness.

Objections to these proposals include Torrey's [10] concern about "psychiatric fascism" as well as the risk of mental health personnel being lured into no-win situations where we assume responsibility for social problems beyond our scope. Another possible disadvantage stems from the potential of self-fulfilling prophecies victimizing some children identified as vulnerable and high-risk. Rosenthal's [20] demonstration that arbitrary labeling of laboratory animals can affect the results of methodologically rigorous experiments because of observer bias undoubtedly has relevance for children as demonstrated by the enhanced school performance of those children who were arbitrarily identified as fast learners in a comparable experiment. It is assumed 
that the opposite occurs with regularity in nonexperimental situations.

Perhaps the most significant source of difficulty is society and its leaders' typical failure to establish meaningful priorities when faced with the multitude of potential problems and crises to be addressed and hopefully prevented by anticipatory planning. But, in the absence of longer-term planning, major problem-solving programs are started and then canceled with only a minimum of interpretable evidence regarding effectiveness. This suggests that we need new means of reconciling our commitment to democratic decision making with society's need for reliable data and conclusions. To enhance this sort of social reality testing, Campbell [21] has suggested an "experimenting society" with priorities determined by an auxiliary legislature of those empirical social scientists who are program evaluation experts, each of whom shall have been appointed by an elected legislator.

\section{Third Level of Confidence in which Maximal Controversy}

Far Exceeds the Minimal Evidence

Probably the most prominent and in many respects the most controversial issue in prevention is the existence of a variety of childrearing practices that presumably affect child development. The earlier confidence of many advocates of the mental hygiene movement that there was a specific way to raise children "hygienically" to avoid mental illness is no longer as evident; nevertheless, there persists a widespread belief that there are deleterious modes of feeding, toilet training, disciplining children, and so forth, that can contribute to difficulties later in life. Even in the most extreme instances (where the issue is not if the feeding, for example, is being carried out appropriately but rather if it is a vehicle for cruelty) it is still difficult to state with any certainty that there is a relationship between actual practices and subsequent outcomes because of the multitude of other factors that merit consideration. A longitudinal perspective is offered by Wolfenstein's [22] serial review of the Children's Bureau's publication Infant Care through various editions. Reading her summary of the ebbs and tides of various child-rearing recommendations is sobering, to say the least. In fact, there may be cause for concern even in those rare instances in which there has been a consistent trend throughout all editions of this publication, such as the consistent tendency toward liberalization of advice about masturbation, even though the consequences of urging these new recommendations on parents who themselves have been nurtured differently are not known. Is there 
evidence that the underlying attitudes of parents and teachers have in fact changed? How much do we know, for instance, about the extent to which emotional conflicts, presumably related to masturbation, stem from its actual occurrence or from the attendant fantasies?

The preventive payoff from mental health professionals teaching and consulting with the so-called gatekeepers in the community and front-line professionals is difficult to demonstrate, although it seems logical to assume that such cooperation should enable teachers, nonpsychiatric physicians, police, clergy, mental health paraprofessionals, and others to fulfill some aspects of their responsibilities more expertly. Presumably, such activities should result in some promotion of mental health and facilitate early intervention in mental illness. Controversy around this issue tends to be minimal except when it focuses on the question of how much time the mental health professional should devote to such educational-consultative efforts and how much to direct clinical services.

The controversy increases considerably, however, when the problems of educating and consulting with politicians are addressed. Then there is a blurring of the boundaries between our professional-scientific expertise and responsible, active citizenship. The complexities of this interrelationship can become embarrassing when we recall how many of our psychiatric colleagues apparently failed to distinguish their personal political opinions from their professional expertise when they responded to a magazine poll in 1964 ostensibly assessing the mental stability of a presidential candidate whom they had never seen professionally.

Nonetheless, there is the valid question of how we can influence the political process that has such a major influence on clinical psychiatric activities and on preventive programs. Specifically, our preventive focus raises more difficult questions. For example, with over $40 \%$ of the nation's mothers working outside of the home and $10 \%$ of the mothers of children under 6 being the sole support of the family, we must enlist the aid of politicians so that day care will be developmental and not custodial. Politicians are needed also to enable those creative and talented people, who know how to make media presentations attractive enough to be watched, to employ their talents so that television is something other than the vast wasteland that it is so often. Thus, television could have many more programs attractive enough to do well in the ratings, but at the same time would enrich all age levels, whether toddlers, school-aged children, adolescents anticipating parenthood, parents, or potential retirees. Politicians are needed also to redress a situation where such a large percentage of 
the funds for educating children is determined by a direct vote of the taxpayer in contrast to the typical method whereby elected officials determine how much taxpayers will be taxed to support other programs.

In addition to these sticky issues relating to the political process, there is additional controversy, although minimal evidence, about the worthwhileness of enrichment programs, such as: Head Start; family life education employing outreach activities, such as pupils helping pupils and adolescents involved in infant care; Sesame Street; Mister Rogers Neighborhood; those audiovisual aides like Inside-Out designed to teach children about their own emotions; and so many more that seem worthwhile, although there is little supportive evidence.

\section{Discussion}

These blends of clinical-developmental knowledge with massive social action are inevitably disquieting. In the global social arena, there may be a tendency to assume and thereby implicitly teach that correlation represents causality. In consequence, the high correlation between urban slums, delinquency, and mental illness tends to lead us to the assumption that one causes the other. In reality, the statistical fact that there is more schizophrenia in the lower social strata does not prove that schizophrenia stems in part from socioeconomic privation, any more than it proves the hypothesis of a downward drift, that is, that the symptoms of the psychiatric disability generate the lower socioeconomic status. In the clinical microcosm, an opposite tendency can be seen that encourages us to de-emphasize the causal significance of correlations. Therefore, we tend to assume and teach, for instance, that the correlation of marital difficulties with strife about money should not lead to assumptions that one necessarily causes the other.

The simplest solution to the dilemma is the cautious one of avoiding all risk of confusing professional expertise with our role as concerned citizens. Really, many of these issues are our business as citizens, but are they any more so than electoral reform, inflation, pollution, public transportation? It is exceedingly difficult to answer the question as to whether such matters are our business as professionals, so we will close by asking analogous questions as to whether contaminated water is the business of the bacteriologist, leaded paint the business of the pediatrician, unimmunized children the business of the virologist, nuclear fallout the business of the physicist, and so on.

Who else is better equipped, for instance, to help mankind realize 
that the ubiquitous intergenerational conflict readily observed today, in the recent past, and also described in antiquity may represent something more than each succeeding younger generation being irresponsible or ungrateful or rebellious or whatever is currently fashionable to call "them." Could not a good part of this persistent strife that has endured through the ages be stimulated by the older generation's unconscious resentment against the young, who since their birth have been inadvertently reactivating, for their parents, those unconscious developmental conflicts that the parents had most hoped they had long resolved and forgotten? Subsequently, the young are free with their criticisms as they prepare to replace and displace their elders, who bitterly resent growing old. Maybe it would not make any difference if people understood the reasons for intergenerational conflict, but of course nobody will ever know if we do not start trying.

Even if such disparate, wide-sweeping idealistic notions were taught in some systematic didactic fashion in residency programs, would we have any reason to believe in the absence of meaningful experiential activities that it would have any substantial effect on the field other than to state once again that prevention should be an ultimate goal in child psychiatry? If our survey demonstrated anything, it is that at present minimal attention if not lip service is paid to prevention in the education of child psychiatrists. Perhaps meetings of program directors should be devoted to generating ideas about how we can raise the status of prevention at least on a par with the five functions of the child psychiatrist highlighted in the 1963 conference. Perhaps funding might be sought to encourage each residency program in child psychiatry to develop, as a part of its program, a specific, focused, testable preventive project. More modestly, programs might require their residents to indicate on each clinical chart how they think the problem might have been prevented. There are a myriad of other possible ideas, and our challenge is not only to generate and disseminate them but also to translate them into fruitful action.

Perhaps the ambiguous status of prevention in child psychiatric education is partly an inevitable consequence of the fragmentation of child psychiatric services, which in turn is reflected in the patterns of child psychiatric residency education. To what extent does the educational core, emphasized in child psychiatric residencies, truly serve as an educational foundation on which the resident subsequently can build in any of a variety of directions? How much does it concentrate on training in specific circumscribed skills and thereby strongly influence the directions in which the child psychiatrist develops over the 
course of his or her career, which in turn is reflected in the unevenness and fragmentation of a child psychiatric service delivery, which in turn is reflected in residency education?

Clearly, no residency program could provide each resident with meaningful educational experiences in all child psychiatric services. Nevertheless, would it not be ideal if residency programs (not the individual residents) were part of a model comprehensive interrelated network of services that included all those services required by the community at the then current level of knowledge-ranging from primary prevention at one end to rehabilitation at the other end of the continuum, without neglecting the many types of services required in between these two. To be meaningful and not a fraud, this model program should service a small finite community (that, for educational purposes, would ideally include inner-city, suburban, and rural representation, yet at the same time be small enough) so this ideal model program could truly understand the community and be responsive to its changing needs as well as to our expanding knowledge. If such an ideal model could be established, there would be many levels at which educational programs could tap in. A significant part of the exposure would include assessment of effectiveness as well as opportunities to observe and study the evolutionary process of change in a comprehensive service delivery system endeavoring to increase effectiveness and responsiveness to an ever-changing social reality.

Obviously, such ideal proposals are as easy to dismiss as unrealistic "pie in the sky" as they are to propose. Both monetary and personnel resources are not available, and we should add that they probably never will be if we do not endeavor to establish such models with the conviction that the tremendous expense may eventually prove worthwhile. Otherwise what we try to do in child psychiatric education and service may continue to have the feel of sweeping back the ocean with a broom.

\section{References}

1. Crutcher R: Child psychiatry: A history of its development. Psychiat 6:191201, 1943.

2. Lowrey L: Psychiatry for children: A brief history of developments. Amer $J$ Psychiat 101:375-388, 1944.

3. Kanner L: Child Psychiatry (4th ed). Springfield, Ill, Charles C Thomas, 1972.

4. Gardner G: History of child psychiatry. In AM Freedman, HI Kaplan \& BJ Sadock (Eds), Comprehensive Textbook of Psychiatry (2nd ed). Baltimore, Williams \& Wilkins, 1975. 
5. Krug O, et al (Eds): Career Training in Child Psychiatry (Report of the Conference on Training in Child Psychiatry, January 10-15, 1963). Washington, DC, American Psychiatric Association, 1964.

6. Caplan G: Principles of Preventive Psychiatry. New York, Basic Books, 1964.

7. Caplan G: Recent trends in preventive child psychiatry. In G Caplan (Ed), Emotional Problems of Early Childhood. New York, Basic Books, 1955.

8. Anthony EJ: Research as an academic function of child psychiatry. Arch Gen Psychiat 21:385-391, 1969.

9. Mead M: A Way of Seeing. New York, William Morrow, 1974.

10. Torrey EF: The Death of Psychiatry. Philadelphia, Chilton, 1974.

11. Spitz R: Hospitalism. Psychoanal Study of the Child 1:53-74, 1945.

12. Bowlby J: Maternal Care and Mental Health (Monogr 2). Geneva, World Health Organization, 1951.

13. Goldfarb W: Emotional and intellectual consequences of psychological deprivation in infancy: A reevaluation. In PH Hoch \& J Zubin (Eds), Psychopathology of Childhood. New York, Grune \& Stratton, 1955.

14. Call J: Some problems and challenges in the geography of scholarship for child psychiatry. J Amer Acad Child Psychiat 15:139-160, 1976.

15. Klaus M, Kennell J: Mothers separated from their newborn infants. Pediat Clin North Amer 17, 1970.

16. Pasamanick B, Knobloch H: Epidemiologic studies on the complication of pregnancy and the birth process. In G Caplan (Ed), Prevention of Mental Disorders in Children. New York, Basic Books, 1961.

17. Davie R, Butler N, Goldstein H: From Birth to Seven: Report of the National Child Development Study. London, Longman Group, 1972.

18. Joint Commission on the Mental Health of Children: Crisis in Child Mental Health: Challenge for the 1970's. New York, Harper \& Row, 1969.

19. McDermott W: Medical distribution and modification of disease patterns. Amer J Psychiat 122:1398-1406, 1966.

20. Rosenthal R: Experimental Effects in Behavioral Research. New York, Appleton-Century-Crofts, 1966.

21. Campbell DT: Methods for the experimenting society. Amer Psychol, in press.

22. Wolfenstein M: Trends in infant care. Amer J Orthopsychiat 23:120-130, 1953. 